\title{
A case of lymph node metastasis following a curative endoscopic submucosal dissection of an early gastric cancer
}

\author{
Hisaharu Oya - Takuji Gotoda - Tetsu Kinjo • Haruhisa Suzuki • \\ Shigetaka Yoshinaga $\cdot$ Hirokazu Taniguchi $\cdot$ Ryoji Kushima $\cdot$ Makoto Saka \\ Hitoshi Katai • Ichiro Oda
}

Received: 26 April 2011/Accepted: 19 October 2011/Published online: 15 November 2011

(C) The International Gastric Cancer Association and The Japanese Gastric Cancer Association 2011

\begin{abstract}
Currently in Japan, differentiated gastric submucosal invasive cancers $<500 \mu \mathrm{m}$ (SM1) with negative lymphovascular involvement are included in expanded pathological criteria for curative endoscopic treatment. This is based on a retrospective examination of surgical resection cases in which patients suitable for such expanded criteria were determined to have a negligible risk of lymph node metastasis. We performed endoscopic submucosal dissection on a 65-year-old male with early gastric cancer in April 2005, and pathology revealed a welldifferentiated adenocarcinoma, $21 \times 10 \mathrm{~mm}$ in size, SM1 invasion depth and negative lymphovascular invasion as well as tumor-free margins, so the case was diagnosed as a curative resection. This case, however, resulted in lymph node metastasis that was diagnosed by endoscopic ultrasonography with fine-needle aspiration biopsy in May 2009. Distal gastrectomy with D2 lymph node dissection
\end{abstract}

H. Oya $\cdot$ T. Gotoda $\cdot$ T. Kinjo $\cdot$ H. Suzuki .

S. Yoshinaga $\cdot$ I. Oda $(\bowtie)$

Endoscopy Division, National Cancer Center Hospital,

5-1-1 Tsukiji, Chuo-ku, Tokyo 104-0045, Japan

e-mail: ioda@ncc.go.jp

T. Gotoda

Department of Gastroenterology and Hepatology,

National Center for Global Health and Medicine, Tokyo, Japan

H. Taniguchi · R. Kushima

Pathology Division, National Cancer Center Hospital,

Tokyo, Japan

M. Saka · H. Katai

Gastric Surgery Division, National Cancer Center Hospital,

Tokyo, Japan was then performed, confirming lymph node metastasis from the original gastric cancer.

Keywords Early gastric cancer · Lymph node metastasis · Expanded criteria $\cdot$ ESD $\cdot$ SM1

\section{Introduction}

Endoscopic resection is the preferred treatment method for the local dissection of early cancer with a negligible risk of lymph node metastasis. For early gastric cancer (EGC), it is possible to achieve nearly $100 \%$ curability by radical surgery; therefore, it is an absolute requirement to maintain such a level of curability with endoscopic resection. In Japan, the Gastric Cancer Treatment Guideline (3rd version) specifies that the pathological criteria for curative endoscopic resection are limited to small intramucosal differentiated-type gastric cancer $\leq 20 \mathrm{~mm}$ in size without an ulcer finding [1]. Recently, the pathological criteria for curative endoscopic resection of EGC have been expanded to cover other lesions with a negligible risk of lymph node metastasis [2]. These expanded criteria include larger lesions, lesions with ulceration and lesions that invade the submucosa $<500 \mu \mathrm{m}$ (SM1).

Together with advances in treatment equipment used for endoscopic resection in recent years, endoscopic submucosal dissection (ESD) provides a higher en bloc resection rate, thus allowing for more accurate and detailed pathological evaluation compared to endoscopic mucosal resection (EMR) [3-8]. Due to the refinement of ESD and the acceptance of the expanded pathological criteria for curative endoscopic resection, which in turn resulted in expanded clinical indications for endoscopic resection, the 
number of patients who are treated by endoscopic resection has increased dramatically.

We experienced for the first time a case of lymph node metastasis following ESD resulting in a pathologically curative resection of SM1 EGC and report this case here.

\section{Case report}

A 65-year-old man was admitted in 2005 because EGC was detected by screening endoscopy. There were no abnormalities in the physical examination or laboratory data, and no medical history of malignant tumors. The lesion was a type 0-IIc well-differentiated adenocarcinoma, $20 \mathrm{~mm}$ in size with an ulcer scar on the anterior wall near the pylorus (Fig. 1a, b). ESD was performed on this lesion, resulting in an en bloc resection. Precise pathological examination of 2-mm slices from the entire specimen revealed a type 0 -IIc lesion $21 \times 10 \mathrm{~mm}$ in size with an ulcer scar that was predominantly a well-differentiated adenocarcinoma with an intramucosal, but not a submucosal poorly differentiated adenocarcinoma component in the proximal portion of the lesion. The poorly differentiated adenocarcinoma component was separate from the ulcer scar. The tumor involved SM1 invasion with negative lymphovascular involvement and horizontal/vertical margins, so it was determined that the resection was curative based on the expanded pathological criteria (Fig. 2a-c). Thereafter, we performed computed tomography (CT) or endoscopic ultrasonography (EUS) on an alternating basis every 6 months as well as endoscopy every year. In December 2007, CT detected an enlarged lymph node near the pylorus $(12 \times 9 \mathrm{~mm})$ (Fig. 3), and the laboratory data indicated an increase in CEA of $12.4 \mathrm{ng} / \mathrm{ml}$ (normally $<5.0 \mathrm{ng} / \mathrm{ml}$ ). EUS was unable to detect the enlarged lymph node, so we continued EUS follow-up every 2 or 3 months. In July 2008, EUS revealed a 12-mm enlarged lymph node at the same site indicated by CT in December 2007. This enlarged lymph node had some highly echoic spots, so it was thought to be an inflammatory reaction, and we continued EUS followup every 2 or 3 months. Although the size of the lymph node did not change, a new 6-mm enlarged lymph node near the original site was detected by EUS in May 2009 (Fig. 4a), and the CEA level had increased to $17.5 \mathrm{ng} / \mathrm{ml}$ by then. For diagnosis of the enlarged lymph nodes, EUS with fine-needle aspiration biopsy (EUS-FNA) was carried out, and a pathological examination revealed an adenocarcinoma (Fig. 4b). No other lesions were detected; therefore, lymph node metastasis was diagnosed as resulting from the SM1 EGC originally treated by ESD. In July 2009, a distal gastrectomy with D2 lymph node dissection was carried out, and postoperative pathological examination revealed no local recurrent tumor at the ESD site in the stomach. Metastasis was limited to the lymph node near the pylorus (Fig. 5a, b). No recurrence has been subsequently detected following the gastrectomy.

\section{Discussion}

We previously reported a group of patients with negligible risk of lymph node metastasis based on the pathological examination of over 5,000 surgical EGC cases that developed into the current expanded pathological criteria for curative endoscopic resection of EGC [2]. The expanded criteria include differentiated adenocarcinomas with negative lymphovascular involvement that are either intramucosal cancer without ulcer findings regardless of tumor size, intramucosal cancer with ulcer finding $\leq 3 \mathrm{~cm}$ in size
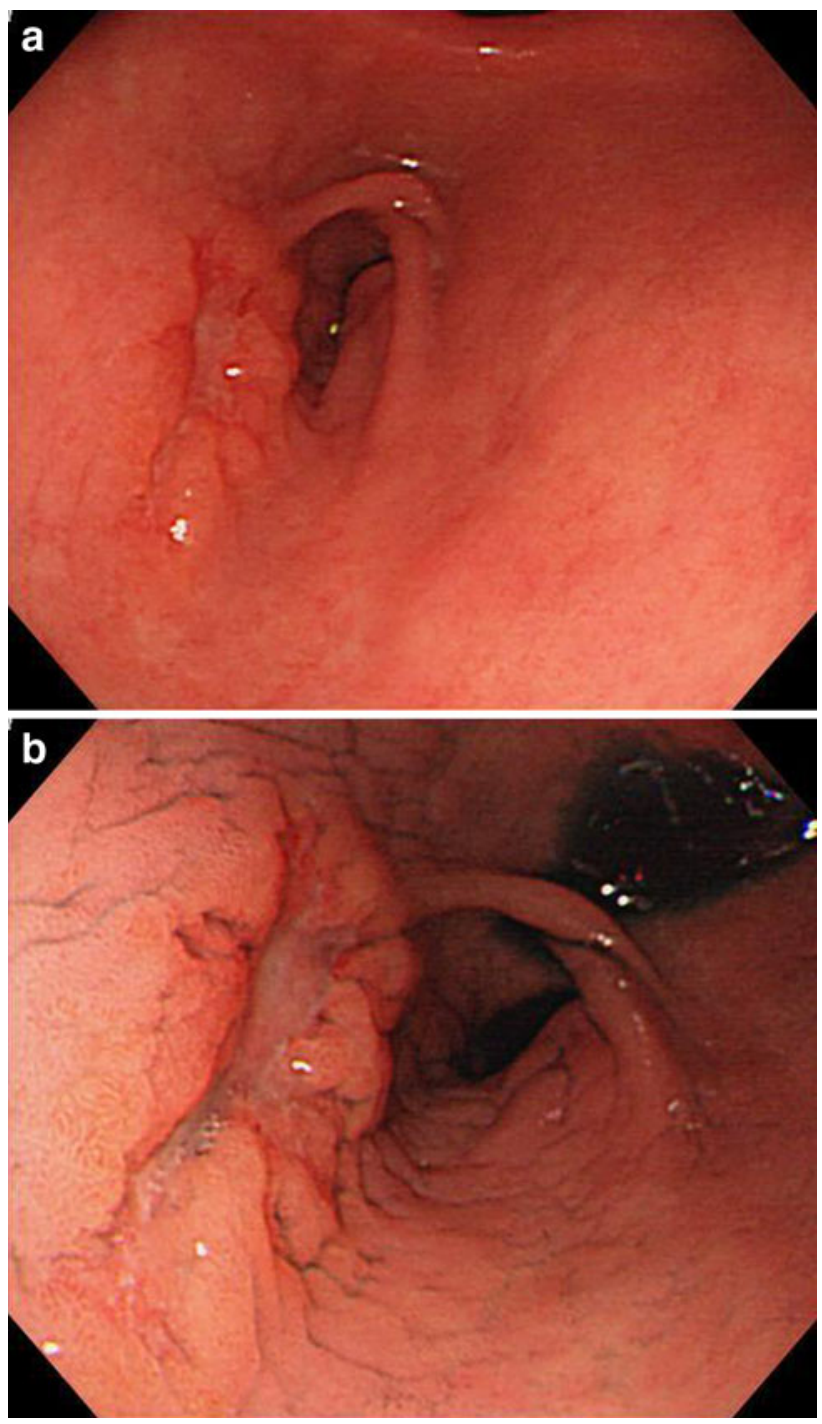

Fig. 1 a Endoscopy detected a type 0-IIc lesion $20 \mathrm{~mm}$ in size with an ulcer scar on the anterior wall near the pylorus. b Chromoendoscopy using indigo-carmine clearly revealed the margin of the lesion 

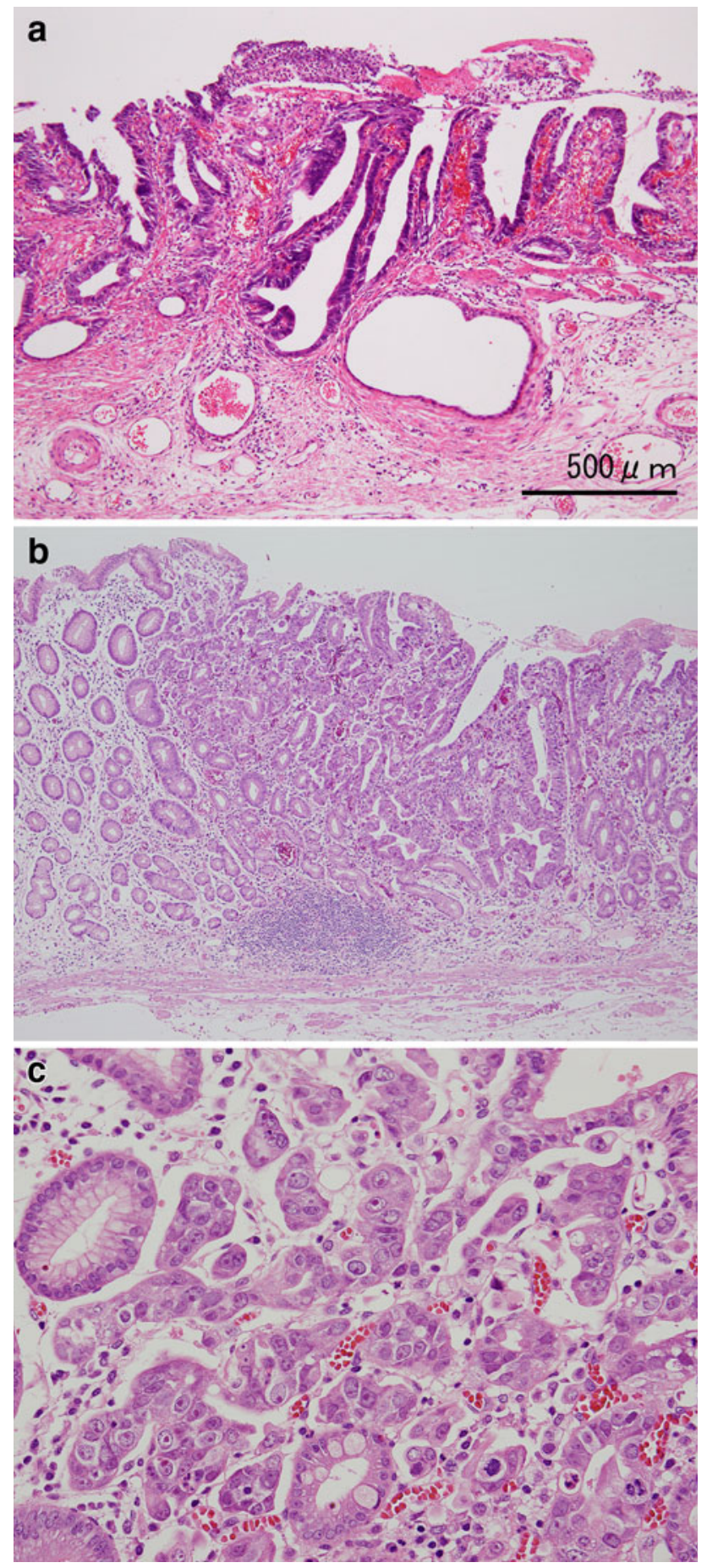

Fig. 2 a Pathological examination revealed a type 0-IIc lesion with ulcer scar, $21 \times 10 \mathrm{~mm}$ in size, that had invaded $<500 \mu \mathrm{m}$ with negative lymphovascular involvement and horizontal/vertical margins. b, c Resected specimen predominantly revealed a well-differentiated adenocarcinoma with a poorly differentiated adenocarcinoma component in just the intramucosal proximal portion of the lesion

or SM1 cancer $\leq 3 \mathrm{~cm}$ in size. Patients who were treated following these expanded criteria have had similar longterm outcomes to those treated according to traditional

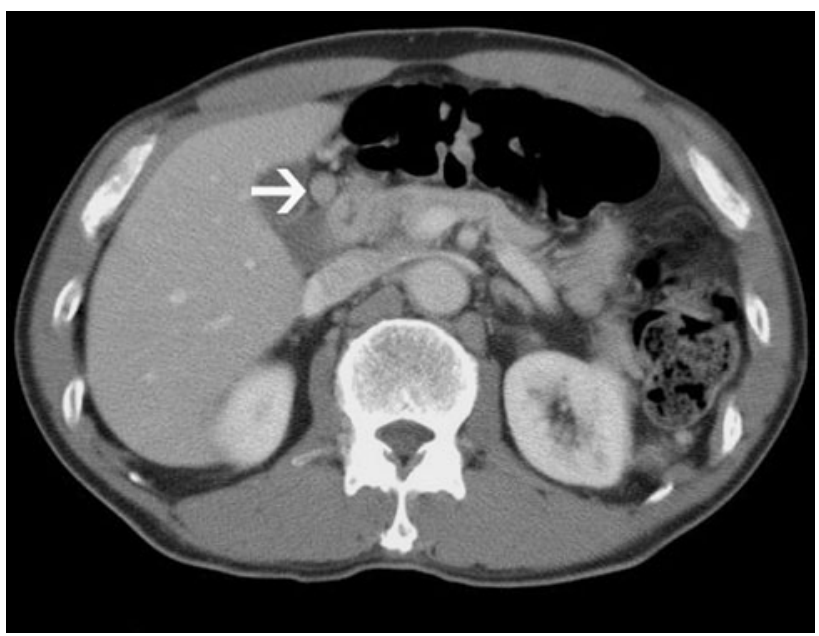

Fig. 3 CT in December 2007 detected an enlarged suprapyloric lymph node measuring $12 \times 9 \mathrm{~mm}$ in size
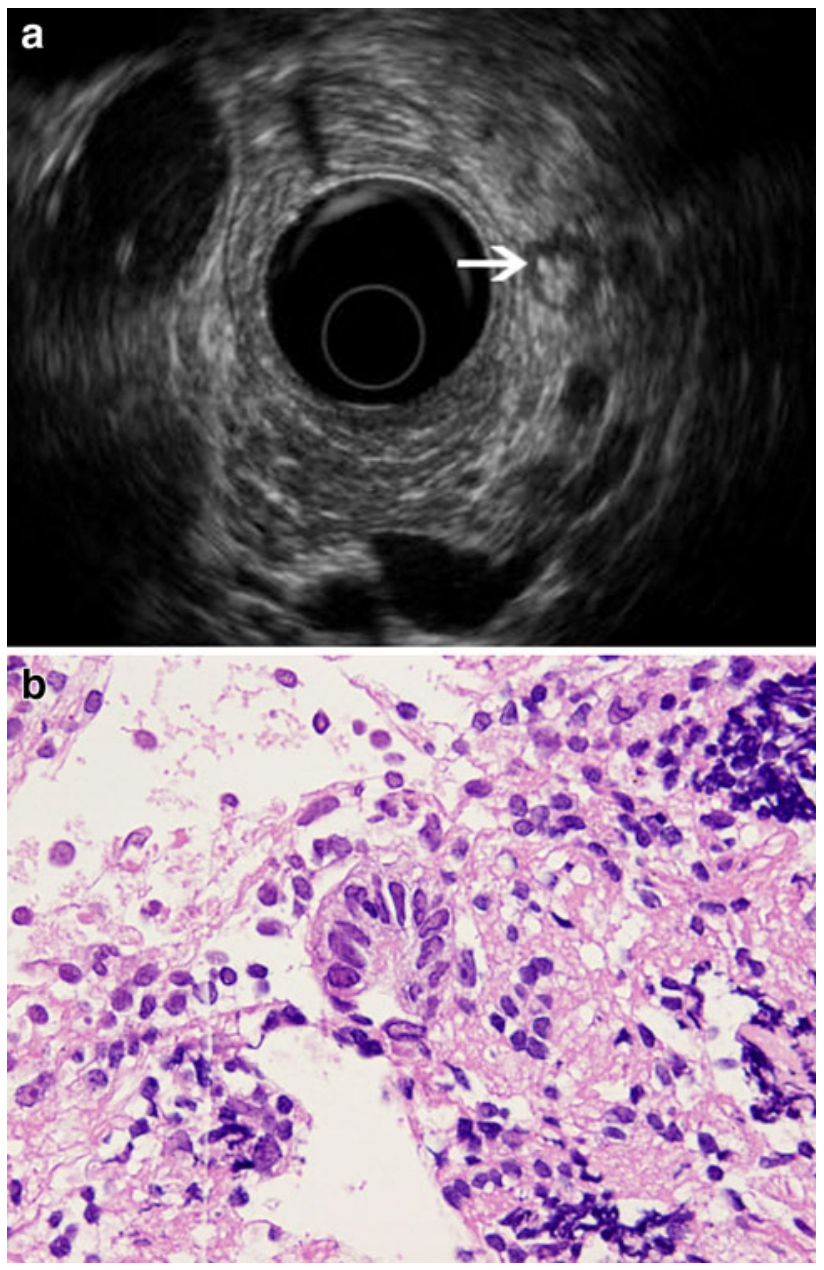

Fig. 4 a EUS in May 2009 revealed an enlarged lymph node measuring $12 \mathrm{~mm}$ in size at the site previously revealed by CT as well as another lymph node measuring $6 \mathrm{~mm}$ in size at a site close to the first one. b An adenocarcinoma with an enlarged nucleus and border irregularities was pathologically revealed by EUS-FNA 

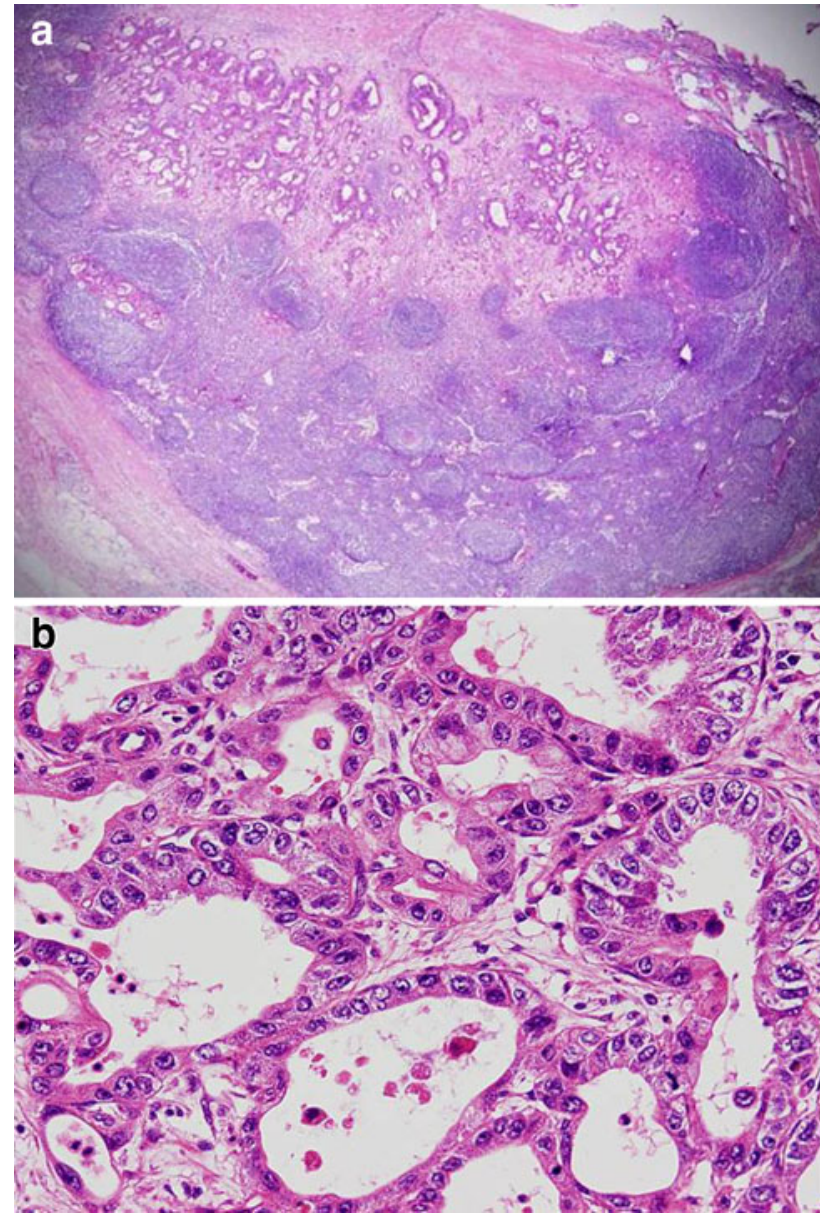

Fig. 5 Lymph node resected by surgery revealed a well-differentiated adenocarcinoma

guideline criteria (5-year survival rate of expanded criteria vs. traditional guideline criteria: 93.4 vs. 92.4\%) [9]. In recent years, Nagano et al. [10] reported two cases of lymph node metastasis of SM1 cancer; however, one patient was previously treated by endoscopic piecemeal resection, and submucosal lymphatic involvement was observed in the other case. No lymphovascular involvement was observed in the present case, however, and this is the first case of a curative resection based on the SM1 expanded criteria in which lymph node metastasis was observed during careful follow-up.

We speculate that there are two possible explanations for such lymph node metastasis. First, we have previously reported that SM1 gastric cancer differentiated adenocarcinomas with negative lymphovascular involvement $\leq 3 \mathrm{~cm}$ in size had a $0 \%$ rate $(0 / 145)$ of lymph node metastasis with a $95 \%$ confidence interval (CI) upper limit of $2.5 \%$ [2]. The risk of lymph node metastasis for such SM1 gastric cancer is slightly higher, however, than for the expanded criteria for intramucosal cancer. Second, the resected specimen revealed a predominantly well- differentiated adenocarcinoma, but a poorly differentiated adenocarcinoma component also was identified in the proximal portion of the lesion, although it was not in the area of SM1 infiltration and separate from the ulcer scar. A similar case of lymph node metastasis following ESD has been reported involving such a mixed type adenocarcinoma, and the patient subsequently died of liver metastasis 33 months after ESD [11].

In our case, lymph node metastasis was pathologically confirmed by EUS-FNA, and radical surgical treatment was performed, thus far resulting in the patient having no recurrence. Needless to say, careful follow-up is necessary after endoscopic resection, but it is difficult for current diagnostic imaging equipment to differentiate whether slightly enlarged lymph nodes, as in the present case, are non-malignant or metastatic [12]. Iwashita et al. [13] analyzed the enlarged lymph nodes of 62 patients and reported EUS-FNA sensitivity to be $97 \%$ with a specificity of $100 \%$. EUS-FNA was also effective in the present case; therefore, we believe that EUS-FNA can be of assistance in making an accurate diagnosis for patients with enlarged lymph nodes.

We have to maintain careful follow-up, keeping in mind that metastasis may possibly occur even in a case of curative resection based on the expanded pathological criteria.

\section{References}

1. Japanese Gastric Cancer Association. Treatment guidelines for gastric cancer in Japan. 3rd ed. Tokyo: Kanehara; 2010 (in Japanese).

2. Gotoda T, Yanagisawa A, Sasako M, Ono H, Nakanishi Y, Shimoda $\mathrm{T}$, et al. Incidence of lymph node metastasis from early gastric cancer: estimation with a large number of cases at two large centers. Gastric Cancer. 2000;3:219-25.

3. Gotoda T, Yamamoto H, Soetikno R. Endoscopic submucosal dissection of early gastric cancer. J Gastroenterol. 2006;41:929-42.

4. Oda I, Gotoda T, Hamanaka H, Eguchi T, Saito Y, Matsuda T, et al. Endoscopic submucosal dissection for early gastric cancer: technical feasibility, operation time and complications from a large consecutive series. Dig Endosc. 2005;17:54-8.

5. Yamamoto H, Kawata H, Sunada K, Sasaki A, Nakazawa K, Miyata T, et al. Successful one-piece resection of large superficial tumors in the stomach and colon using sodium hyaluronate and small-caliber-tip transparent hood. Endoscopy. 2003;35:690-4.

6. Oyama T, Kikuchi Y. Aggressive endoscopic mucosal resection in the upper GI tract-hook knife EMR method. Minim Invasive Ther Allied Technol. 2002;11:291-5.

7. Fujishiro M, Yahagi N, Nakamura M, Kakushima N, Kodashima $\mathrm{S}$, Ono S, et al. Successful outcomes of a novel endoscopic treatment for GI tumors: endoscopic submucosal dissection with a mixture of high-molecular-weight hyaluronic acid, glycerin, and sugar. Gastrointest Endosc. 2006;63:243-9.

8. Oda I, Saito D, Tada M, Iishi H, Tanabe S, Oyama T, et al. A multicenter retrospective study of endoscopic resection for early gastric cancer. Gastric Cancer. 2006;9:262-70. 
9. Gotoda T, Iwasaki M, Kusano C, Seewald S, Oda I. Endoscopic resection of early gastric cancer treated by guideline and expanded National Cancer Centre criteria. $\mathrm{Br}$ J Surg. 2010;97:868-71.

10. Nagano H, Ohyama S, Fukunaga T, Hiki N, Seto Y, Yamaguchi $\mathrm{T}$, et al. Two rare cases of node-positive differentiated gastric cancer despite their infiltration to sm1, their small size, and lack of lymphatic invasion into the submucosal layer. Gastric Cancer. 2008;11:53-8.

11. Hanaoka N, Tanabe S, Higuchi K, Sasaki T, Nakatani K, Ishido $\mathrm{K}$, Ae T, Koizumi W, Saigenji K, Mikami T. A rare case of histologically mixed-type intramucosal gastric cancer accompanied by nodal recurrence and liver metastasis after endoscopic submucosal dissection. Gastrointest Endosc. 2009;69:588-90.

12. Kwee RM, Kwee TC. Imaging in assessing lymph node status in gastric cancer. Gastric Cancer. 2009;12:6-22.

13. Iwashita T, Yasuda I, Doi S, Nakashima M, Thurumi H, Hirose $\mathrm{Y}$, et al. Endoscopic ultrasound-guided fine-needle aspiration in patients with lymphadenopathy suspected of recurrent malignancy after curative treatment. J Gastroenterol. 2009;44:190-6. 